\title{
Adversidad psicosocial, psicopatología y funcionamiento en hermanos adolescentes en alto riesgo (HAR) con y sin trastorno por déficit de atención con hiperactividad (TDAH)
}

\author{
Lino Palacios-Cruz, ${ }^{1,3}$ Adriana Arias-Caballero, ${ }^{3}$ Rosa Elena Ulloa, ${ }^{4}$ Norma González-Reyna, ${ }^{4}$ \\ Pablo Mayer-Villa, ${ }^{3}$ Miriam Feria, ${ }^{2,3}$ Liz Sosa, ${ }^{2}$ Francisco R. de la Peña, ${ }^{2}$ Alfonso Cabrera-Lagunes, ${ }^{5}$ \\ Alejandra Fragoso, ${ }^{6}$ Alfredo Sepúlveda, ${ }^{6}$ Carlos S. Cruz, ${ }^{7}$ Patricia Clark Peralta, ${ }^{8}$ Juan C. Pérez, ${ }^{9}$ \\ María C. Lara-Muñoz, ${ }^{10}$ Corina Benjet ${ }^{11}$
}

Artículo original

\section{SUMMARY}

Introduction

Attention deficit hyperactivity disorder (ADHD) is a highly heritable neurodevelopmental disorder. The determination of the clinical features, adversity factors and level of functioning in siblings of probands with ADHD, known as a high risk (ADHD R Sib), could help us to establish the risk to which they are subjected.

\section{Objective}

To determine the frequency of ADHD and other psychiatric disorders in R Sib. Secondary objectives were to establish the psychosocial adversity factors that predict ADHD R Sib and determine the magnitude of effect on performance and other psychiatric disorders when siblings have ADHD (R Sib +) compared to those without ADHD (R Sib -).

\section{Material and methods}

This multicenter study is descriptive, transversal and analytical. The sample $(n=84)$ was formed by adolescent siblings of probands with ADHD who shared both parents.

\section{Results}

While 45.2\% ( $n=38)$ had ADHD, $17.9 \%(n=15)$ had no psychiatric disorder. Being a R Sib + increased at least four times the likelihood of having oppositional defiant disorder (OR=4.3, 95\% $\mathrm{Cl}$ 1.3-14.8). These data remained significant when adjusted for sex, age and number of adversities (RM 95=3, 1.8-10.9\%). Being a R Sib+ increased almost five times the probability of academic dysfunction $(O R=4.84$, $95 \% \mathrm{Cl} 1.41-16.63)$. The overall average was 3.3 adversities $(S D=1.4)$. Psychopathology in both parents was found in $51.2 \%$ of the sample (ES=0.055). Severe family dysfunction increased 2.5 times the probability of having ADHD in a $\mathrm{R}$ Sib $195 \% \mathrm{Cl}, 1.06$ 6.25). When comparing the groups with three or more psychosocial adversities, there were no significant differences $(81.6 \%$ vs. $65.2 \%$, $\mathrm{p}=0.14)$.

\section{Conclusions}

The clinical study of R Sib for ADHD is necessary due to the different implications in terms of prevention, early care and prognosis improvement of these patients.

Key words: High risk siblings, adolescents, ADHD, psychosocial adversity, comorbidity.

\section{RESUMEN}

\section{Introducción}

El trastorno por déficit de atención con hiperactividad (TDAH) es un trastorno del neurodesarrollo altamente heredable. La determinación de las características clínicas, los factores de adversidad y el nivel de funcionamiento en hermanos de probandos con TDAH, denominados en alto riesgo (HAR), podría ayudar a establecer el riesgo al cual están sometidos.

Subdirección de Investigaciones Clínicas, Instituto Nacional de Psiquiatría Ramón de la Fuente Muñiz (INPRFM)

Clínica de la Adolescencia, INPRFM.

Programa de Detección Oportuna de TDAH en el Adulto (PROMETEO), INPRFM

Laboratorio de Psicofarmacología del Desarrollo, Hospital Psiquiátrico Infantil Juan N Navarro (HPIJNN).

Facultad de Medicina, Universidad Veracruzana, Campus Jalapa.

Facultad de Ciencias, Universidad Nacional Autónoma de México.

Laboratorio de Genética Psiquiátrica, INPRFM.

Departamento de Epidemiología Clínica, Hospital Infantil de México Federico Gómez.

Fundación Federico Hoth AC.

Facultad de Medicina, Benemérita Universidad Autónoma de Puebla.

Dirección de Investigaciones Epidemiológicas y Psicosociales, INPRFM.

Correspondencia: Lino Palacios-Cruz. Departamento de Epidemiología Clínica, Subdirección de Investigaciones Clínicas, Instituto Nacional de Psiquiatría Ramón de la Fuente Muñiz, Calz. México-Xochimilco, 101, San Lorenzo Huipulco, Tlalpan, 14370, México, DF. E-mail: lino.palacios@salud-mental.org.mx 


\section{Objetivo}

Determinar la frecuencia de TDAH y otros trastornos psiquiátricos en HAR. Como objetivos secundarios, establecer los factores de adversidad psicosocial que predicen el TDAH en HAR y determinar la magnitud del efecto sobre el funcionamiento y otros trastornos psiquiátricos cuando los hermanos tienen TDAH (HAR TDAH +) comparados con quienes no lo tienen (HAR TDAH -).

\section{Material y método}

Este estudio multicéntrico es descriptivo, transversal y analítico. La muestra $(n=84)$ se conformó por hermanos adolescentes de probandos diagnosticados con TDAH que compartían a ambos padres.

\section{Resultados}

El $45.2 \%(n=38)$ tuvo TDAH. El $17.9 \%(n=15)$ no presentó trastorno psiquiátrico alguno. Ser HAR TDAH+ incrementó al menos cuatro veces más la probabilidad de presentar trastorno negativista desafiante ( $R M=4.3$; IC 95\% 1.3-14.8), dato que mantuvo significan- cia al ajustarse por sexo, edad y número de adversidades ( $R M=3$ IC 95\% 1.8-10.9). Ser HAR TDAH+ incrementó casi cinco veces la probabilidad de presentar disfunción académica (RM=4.84 IC $95 \%$ 1.41-16.63). El promedio general de adversidades fue 3.3 $(D E=1.4)$. Encontramos psicopatología en ambos padres en el $51.2 \%$ de la muestra $(E S=0.055)$. La disfunción familiar grave incrementó 2.5 veces la probabilidad de presentar TDAH en los HAR (IC $95 \%$ 1.06-6.25). Al comparar los grupos con tres o más adversidades psicosociales, no existieron diferencias significativas $181.6 \%$ vs. $65.2 \% ; p=0.14)$.

\section{Conclusiones}

El estudio clínico de los HAR para TDAH es necesario debido a las distintas implicaciones que tiene a nivel de la prevención, la atención oportuna y la mejoría del pronóstico de estos sujetos.

Palabras clave: Hermanos en alto riesgo, adolescentes, TDAH, adversidad psicosocial, comorbilidad.

\section{INTRODUCCIÓN}

El trastorno por déficit de atención con hiperactividad (TDAH) es definido por el DSM- $5^{1}$ como un trastorno del neurodesarrollo, que se inicia regularmente antes de los 12 años de edad y que persiste durante la adolescencia e incluso en la vida adulta. Estudios meta-analíticos y epidemiológicos señalan que al menos el 5.29\% de los niños y adolescentes presenta este trastorno. ${ }^{2-6}$ Estas cifras, además del impacto que genera su tardío reconocimiento, lo colocan como un problema de salud pública a nivel mundial. ${ }^{2}$

Es uno de los trastornos complejos más heredables, con un coeficiente de heredabilidad de $76 \% .^{7}$ En diversos estudios se ha visto que además de la adversidad biológica, existe adversidad psicosocial y que es especialmente la percepción y respuesta individual a factores medioambientales $^{8}$ lo que incrementa la probabilidad, en el inter-juego gen-ambiente, de que los hermanos adolescentes de pacientes con TDAH presenten el mismo diagnóstico. ${ }^{9,10}$ Varios estudios muestran que los sujetos con TDAH, comparados con sujetos controles, se encuentran más expuestos a situaciones estresantes y a factores relacionados con eventos de adversidad psicosocial. ${ }^{11,12}$ La razón de riesgo de recurrencia $(\lambda)$ para TDAH en hermanos es alrededor de $9,,^{13}$ con una mayor cifra $(\lambda=26.2)$ para los hermanos de niños con TDAH y trastornos psiquiátricos comórbidos, comparados con los hermanos de sujetos controles escolares sanos. ${ }^{14}$ Ante este panorama, sabemos que los familiares en primer grado de sujetos con TDAH comparados con familiares de sujetos controles tienen al menos cuatro veces mayor probabilidad de estar afectados. ${ }^{15}$ Lo anterior nos lleva a pensar que estos individuos pertenecen a una población "en alto riesgo". Con base en esto y para fines del presente estudio, a los hermanos de los probandos con TDAH los llamaremos Hermanos en Alto Riesgo (HAR).
Los hallazgos en hermanos de niños y adolescentes con TDAH muestran un nivel intermedio de afectación entre los probandos con el trastorno y los controles sin el trastorno con respecto a los síntomas de TDAH y las condiciones comórbidas. ${ }^{14-17}$ Sin embargo, existen algunos trabajos que han mostrado que los hermanos afectados no referidos son similares a sus hermanos probandos con el mismo diagnóstico y que los hermanos no afectados son similares a los sujetos controles sanos, excepto en la dimensión de problemas emocionales o de trastornos afectivos y ansiosos según sea la visión dimensional o categórica, respectivamente. ${ }^{17,18}$

La determinación de las características clínicas, incluidos los factores de adversidad y el nivel de funcionamiento, relacionadas con los hermanos de probandos con TDAH, podría ayudar al entendimiento de la importancia del riesgo al cual está también sometida esta población y que comparte con el hermano afectado que es llevado a consulta. A nuestro conocimiento, este es el segundo estudio en la literatura especializada en el cual se tiene como objetivo determinar, en hermanos no referidos, la frecuencia de TDAH y de otros trastornos psiquiátricos, además de comparar la relación de distintos factores de adversidad psicosocial y posible afectación en distintas áreas del funcionamiento con el estatus diagnóstico de TDAH entre estos hermanos.

\section{MATERIAL Y MÉTODOS}

Tras la aprobación por los comités de ética correspondientes, se desarrolló el presente estudio en tres centros participantes, dos de ellos fueron instituciones de atención a la salud mental: a) Instituto Nacional de Psiquiatría Ramón de la Fuente Muñiz (INPRFM) y b) Hospital Psiquiátrico Infantil Juan N Navarro (HPIJNN), y el tercero fue en una institución de la sociedad civil dedicada a la orientación y 
educación de pacientes con TDAH y sus familiares: la Fundación Federico Hoth AC (FFHAC). El diseño de este estudio multi-céntrico fue descriptivo, transversal y analítico.

El reclutamiento de los HAR en los centros participantes fue de acuerdo a los siguientes criterios de inclusión: a) adolescentes de 13 a 19 años 11 meses y al menos uno de sus padres, que aceptaron participar por medio de la firma del asentimiento y consentimiento informado, respectivamente, b) de ambos sexos, c) que supieran leer y escribir, d) hermanos biológicos de pacientes adolescentes diagnosticados con TDAH que acudían a las instituciones participantes (HPIJNN, FFHAC o INPRF) y que, e) a juicio del clínico, no tuvieran algún impedimento para participar en la evaluación clínica, como discapacidad intelectual. Se excluyeron sujetos a) que eran producto de un parto gemelar, b) que ya estuvieran recibiendo atención médica o psicológica, c) que estuvieran bajo tratamiento psicofarmacológico, d) que tuvieran alguna enfermedad crónico-degenerativa que comprometiera al Sistema Nervioso Central. Se eliminaron sujetos que 1) no completaran al menos el $75 \%$ de los instrumentos clínimétri$\cos$ y 2) no acudieran a dos citas previamente programadas.

\section{Evaluación clínica}

La evaluación clínica de los adolescentes HAR que participaron en el estudio fue realizada por un profesional en salud mental certificado (psicólogo clínico con maestría o psiquiatra) con al menos cinco años de experiencia clínica. El tiempo total promedio de duración de la evaluación fue de dos horas.

Los profesionales de la salud que participaron en este estudio realizaron el diagnóstico de cualquier trastorno mental de acuerdo a los criterios del DSM-IV y apoyados en la entrevista clínica Brief Psychiatric Rating Scale Child(BPRS- C) versión mexicana de 25 ítems desarrollada en la Clínica de la Adolescencia del INPRFM. Esta versión tiene cuatro reactivos más que la versión original, los cuales evalúan trastornos de la eliminación, hipertimia, uso y abuso de alcohol, tabaco y otras drogas, además de evaluar abuso psicológico y sexual. La confiabilidad ínter evaluador y test-retest es de $r=0.824$ y r=0.661, respectivamente. La entrevista tiene una duración de 30 minutos en promedio, siendo necesario el entrenamiento para su aplicación.

Para cada caso evaluado, la confirmación diagnóstica, incluyendo el TDAH, fue determinada por medio de la revisión y discusión del clínico a cargo de la evaluación y un psiquiatra experto y recertificado, con al menos 15 años de experiencia clínica en salud mental de niños y adolescentes (L.P.C.).

\section{Medición de variables}

A) Gravedad de sintomas del TDAH

Para determinar la gravedad del TDAH se utilizó la versión aplicada por el clínico del Attention Deficit Hiperactivity Dis-or- der Rating Scale (ADHD RS- IV), ${ }^{19,20}$ también llamada escala de DuPaul. Esta escala consta de 18 reactivos que evalúan la presencia y frecuencia de cada uno de los criterios citados en el DSM-IV para el diagnóstico de TDAH. Su confiabilidad ha sido validada y probada en distintas poblaciones a lo largo del mundo. ${ }^{21-23}$ En la aplicación de este instrumento se cuidó especialmente el reporte de la frecuencia de cada conducta siendo para "Nunca", como máximo una vez en seis meses; "Algunas veces", una vez al mes; "Frecuentemente", una vez a la semana y "Muy frecuentemente", dos o más veces a la semana.

Para evaluar la gravedad del resto de la psicopatología de los HAR, se utilizó la versión para padres del Strengths $\mathcal{E}$ Difficulties Questionnaire (SDQ-P; www.sdqinfo.com/). ${ }^{24}$ Este instrumento ha demostrado sus propiedades psicométricas en diferentes culturas con diferentes puntos de corte, ${ }^{25-30}$ y su utilidad cuando se ha aplicado en el contexto clínico $^{30}$ y comunitario. ${ }^{28,29}$

\section{B) Funcionamiento}

La evaluación del funcionamiento en distintas áreas en las que se pueden desempeñar los HAR se realizó por medio de la Weiss Functional Impairment Rating Scale Parent Report (WFIRS-P). ${ }^{31}$ Esta escala fue diseñada para evaluar específicamente el funcionamiento de un individuo con TDAH demostrando ser más sensible para la evaluación de poblaciones afectadas con este trastorno. Es una escala tipo likert cuyos valores para cada reactivo van de cero a tres, evaluando la frecuencia de la dimensión explorada como "nunca o nada en absoluto" hasta "muy frecuentemente o muchísimo". Explora seis dimensiones o áreas: a) familiar, b) aprendizaje y escuela, c) habilidades vitales, d) concepto propio del niño, e) actividades sociales y f) actividades de riesgo. Cada área incluye desde tres hasta 10 reactivos por cada dimensión, siendo en total 50 reactivos. Este instrumento posee una consistencia interna mayor a 0.9 , además de una excelente sensibilidad al cambio en el tiempo y una mayor correlación entre el cambio y la mejoría de los síntomas del TDAH que mediciones como el Children's Global Assessment Scale (CGAS). ${ }^{32}$ Para nuestro estudio, a cada área evaluada se le asignó el estatus de ser "disfuncional" cuando al menos dos de los ítems que conforman cada área eran calificados por la madre o el padre de cada adolescente, con deterioro moderado a severo; es decir con un valor de dos o tres.

\section{C) Adversidad psicosocial}

Cada uno de los factores de adversidad psicosocial fueron preguntados por el clínico y asentados en la "cédula de datos sociodemográficos y adversidad psicosocial" (CEDA-SOCIAL), especialmente diseñada para esta investigación. La CEDA-SOCIAL recaba datos como: a) Edad de inicio del TDAH, b) Número de hijos que integran una familia, considerando cuatro o más hijos como familia grande, c) Condiciones de hacinamiento, d) Discusiones y episodios de violencia física entre los padres, actual y en los últimos seis 
meses, e) Edad de la madre al momento del nacimiento y f) Escolaridad de los padres.

La psicopatología de los padres (es decir tener trastorno depresivo mayor, trastorno de ansiedad generalizada, TDAH y/o trastorno antisocial de personalidad) fue determinada por un evaluador lego independiente, por medio de la entrevista diagnóstica estructurada MINI International Neuropsychiatric Interview (M.I.N.I.); ${ }^{33}$ la cual evalúa los trastornos psiquiátricos más frecuentes según el DSM-IV y la CIE-10; su tiempo de administración aproximado es de 15 minutos. En los estudios de validez y confiabilidad esta entrevista demostró tener un buen comportamiento, comparada con otras entrevistas como la CIDI y una muy alta reproducibilidad inter-evaluador y prueba-reprueba. ${ }^{33,34}$

Con respecto a la disfunción familiar, se le aplicó directamente al adolescente la escala de Apgar familiar. ${ }^{35,36} \mathrm{La}$ discordia marital se determinó confirmando si los padres se encontraban separados más el reporte de discusiones actual y en los últimos seis meses, además de reportar violencia física y de pareja actual y en los últimos seis meses.

\section{Análisis estadístico}

Para los datos descriptivos se utilizaron medidas de tendencia central y de dispersión, específicamente frecuencias, porcentajes y error estándar para variables cualitativas y promedios y desviación estándar para variables cuantitativas. Para el análisis bivariado, en el caso de las variables cualitativas utilizamos ji cuadrada $\left(\chi^{2}\right)$, en tablas de contingencia de $2 \times$ 2 , y la prueba exacta de Fisher cuando la frecuencia por nivel de variable resultó menor a cinco observaciones. En un primer paso del análisis bivariado, quisimos saber si los fac-

Cuadro 1. Datos Sociodemográficos y de Adversidad Psicosocial en hermanos adolescentes en alto riesgo $(\mathrm{HAR}, \mathrm{n}=84)$

\begin{tabular}{|c|c|c|c|c|}
\hline & $X(D E)$ & \multicolumn{2}{|c|}{ Frecuencia $(\%)$} & \multirow[t]{2}{*}{ Error Estándar } \\
\hline Edad & $16.21(2.51)$ & & & \\
\hline \multicolumn{5}{|l|}{ Sexo } \\
\hline Masculino & & 36 & (42.9) & 0.054 \\
\hline \multicolumn{5}{|l|}{ Adolescentes en alto riesgo } \\
\hline - TDAH & & 38 & $(45.2)$ & 0.055 \\
\hline - Subtipo inatento & & 12 & $(31.6)$ & 0.076 \\
\hline - Subtipo mixto & & 26 & (68.4) & 0.076 \\
\hline - Dos o más trastornos psiquiátricos ${ }^{a}$ & & 34 & $(40.5)$ & 0.054 \\
\hline - Número de trastornos psiquiátricos ${ }^{a}$ & $1.5(1.7)$ & & & \\
\hline - Sin trastorno psiquiátrico actual & & 15 & (17.9) & 0.042 \\
\hline \multicolumn{5}{|c|}{ Adversidad psicosocialb } \\
\hline \multicolumn{5}{|l|}{ Características familiares } \\
\hline - Disfunción familiar grave & & 38 & $(45.2)$ & 0.055 \\
\hline - Familia extensa ${ }^{c}$ & & 28 & (33.3) & 0.052 \\
\hline - Discordia marital & & 72 & $(85.7)$ & 0.038 \\
\hline - Hacinamiento en casa & & 16 & $(19.0)$ & 0.043 \\
\hline \multicolumn{5}{|l|}{ Características de los padres } \\
\hline - Ambos padres psicopatología ${ }^{d}$ & & 43 & $(51.2)$ & 0.055 \\
\hline - Ambos padres baja escolaridade & & 57 & (67.9) & 0.051 \\
\hline - Historia de problemas legales & & 10 & (11.9) & 0.036 \\
\hline - Probable trastorno por uso de alcoholf & & 33 & (39.3) & 0.067 \\
\hline - Probable trastorno por uso de sustancias & & 12 & (14.3) & 0.038 \\
\hline - Edad materna de riesgo al momento del nacimientog & & 12 & (14.3) & 0.038 \\
\hline - 3 o más adversidades psicosociales ${ }^{h}$ & & 61 & $(72.6)$ & 0.049 \\
\hline - Número de adversidades psicosocialesh & $3.3(1.4)$ & & & \\
\hline
\end{tabular}

a Otros trastornos psiquiátricos actuales distintos a TDAH.

b Medido por el Formato de Adversidad Psicosocial CEDA-SOCIAL versión clínico.

c Considerando 4 o más hermanos.

d Evaluado a través de la entrevista clínica con apoyo de la entrevista MINI.

e Baja escolaridad se consideró con un grado máximo escolar de Preparatoria o Bachillerato.

$\mathrm{n}=53$.

g Edad materna de riesgo Menor de 19 años o mayor de 35 años.

h Adversidades psicosociales, disfunción familiar, discordia marital, hacinamiento, Baja escolaridad en ambos padres, Psicopatología en ambos padres, Problemas legales en al menos uno de los padres, Edad materna de riesgo al nacimiento y Familia extensa. 


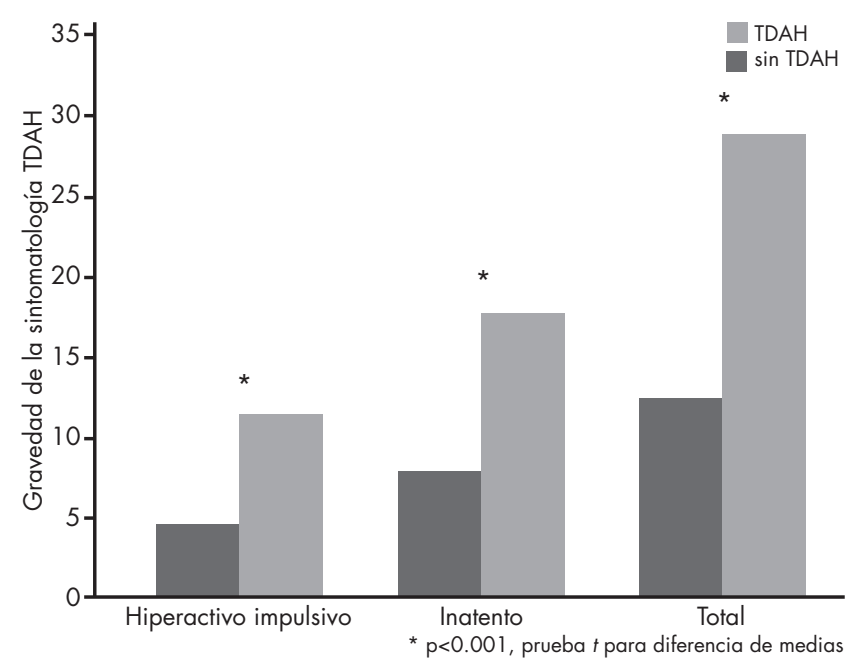

Figura 1. Comparación de la puntuación promedio de la gravedad de TDAH evaluada por el ADHD RS IV en hermanos en alto riesgo con y sin TDAH.

tores de adversidad psicosocial predecían el TDAH en esta muestra de adolescentes, siendo la variable dependiente el estatus diagnóstico de TDAH en los HAR y las variables independientes fueron los factores de adversidad psicosocial. En un segundo paso, la variable TDAH fue asignada como variable independiente y las variables a predecir por separado fueron la presencia o ausencia de disfunción en cada área del funcionamiento y cada uno de los trastornos psiquiátricos comórbidos de acuerdo al DSM-IV.
La variable sexo se utilizó como variable independiente y se asignó el riesgo a ser hombre. Se determinaron las razones de momios no ajustados para cada comparación y posteriormente se obtuvieron las razones de momios ajustadas y su intervalo de confianza al 95\% a partir de regresiones logísticas considerando, además del TDAH, como co-variables, el sexo, la edad y el número de adversidades psicosociales.

Asumiendo una distribución normal, para la comparación entre los HAR TDAH+ y HAR TDAH-, de la edad, la gravedad de los síntomas de TDAH ${ }^{19}$ el número de adversidades psicosociales y el número de trastornos comórbidos, se utilizaron pruebas $\mathrm{T}$ para muestras independientes. Para todas las pruebas se adoptó un nivel alfa de 0.05 . Todos los análisis estadísticos fueron realizados con la Statistical Package for Social Sciences (SPSS), versión 20.

\section{RESULTADOS}

La muestra final de adolescentes en alto riesgo se conformó de 84 sujetos (cuadro 1), el 42.9\% (ES=0.054) fueron hombres. El 53.3\% ( $\mathrm{n}=45)$ de la muestra se reclutó de la clínica de la adolescencia del INPRF, el $41.9 \%(n=35)$ provino del HPIJNN, el $4.8 \%(n=4)$ restante se obtuvo de la FFHAC.

El 45.2\% (ES=0.055) de estos hermanos recibió el diagnóstico de TDAH; de ellos, el 68.4\% (ES=0.076) presentó el subtipo combinado y el resto presentó el subtipo inatento. Arriba del 40\% de los sujetos (ES=0.054) mostró más de dos trastornos psiquiátricos distintos al TDAH. El 17.9\% (ES=0.042) no presentó ningún trastorno psiquiátrico actual.

Cuadro 2. Comparación de las características clínicas y sociodemográficas en Hermanos en alto Riesgo (HAR) con y sin TDAH

\begin{tabular}{|c|c|c|c|c|c|}
\hline & \multicolumn{2}{|c|}{$\mathrm{HAR}(\mathrm{n}=84)$} & \multirow[b]{2}{*}{ Estadístico ${ }^{b, c, d}$} & \multirow[b]{2}{*}{$P$} & \multirow[b]{2}{*}{$\begin{array}{l}\text { RM no ajustado } \\
\text { (IC al } 95 \%)\end{array}$} \\
\hline & $\begin{array}{c}\text { TDAH } \\
\mathrm{n}(\%) / \mathrm{X}(\mathrm{DE}) \\
\end{array}$ & $\begin{array}{c}\text { Sin TDAH } \\
\mathrm{n}(\%) / \mathrm{X}(\mathrm{DE})\end{array}$ & & & \\
\hline Edad & $15.8(2.6)$ & $16.4(2.5)$ & -1.100 & 0.276 & \\
\hline Sexo (Femenino) & $20.0(41.7)$ & $28.0(58.3)$ & 0.460 & 0.510 & $1.40(0.59-3.34)$ \\
\hline \multicolumn{5}{|l|}{ Adversidad psicosociala } & $1.69(0.70-4.07)$ \\
\hline - Disfunción familiar grave* & $22.0(57.9)$ & $16.0(34.8)$ & 4.487 & 0.048 & $2.58(1.06-6.25)$ \\
\hline - Familia extensa & $16.0(42.1)$ & $12.0(26.1)$ & 2.400 & 0.164 & $2.06(0.82-5.18)$ \\
\hline - Discordia marital & $34.0(89.5)$ & $38.0(82.6)$ & 0.801 & 0.533 & $1.78(0.49-6.48)$ \\
\hline - Hacinamiento en casa & $10.0(26.3)$ & $6.0(13)$ & 2.370 & 0.165 & $2.38(0.78-7.31)$ \\
\hline - Ambos padres con psicopatología & $21.0(55.3)$ & $22.0(47.8)$ & 0.461 & 0.519 & $1.35(0.57-3.19)$ \\
\hline - Ambos padres con baja escolaridad & $27.0(71.1)$ & $30.0(65.2)$ & 0.325 & 0.643 & $1.31(0.52-3.31)$ \\
\hline - Historia de problemas legales paterno & $6.0(15.8)$ & $4.0 \quad(8.7)$ & - & 0.337 & $1.97(0.51-7.57)$ \\
\hline - Probable trastorno por uso de alcohole & $15.0(62.5)$ & $18.0(62.1)$ & 0.001 & 1.000 & $1.02(0.33-3.11)$ \\
\hline - Probable trastorno por uso de sustancias & $7.0(18.4)$ & $5.0(10.9)$ & 0.969 & 0.363 & $1.85(0.54-6.39)$ \\
\hline $\begin{array}{l}\text { - Edad de riesgo materno al momento del } \\
\text { nacimientof }\end{array}$ & $6.0(15.8)$ & $6.0(13)$ & 0.128 & 0.762 & $1.25(0.37-4.25)$ \\
\hline - 3 o más adversidades psicosociales & $31.0(81.6)$ & $16.0(65.2)$ & 2.802 & 0.140 & $2.36(0.85-6.55)$ \\
\hline \multicolumn{6}{|c|}{$\begin{array}{l}\text { a Medido por el instrumento CEDA -SOCIAL versión clínico. } \\
\text { b Chi-cuadrada de Pearson. } \\
\text { c Prueba exacta de Fisher (se aplicó en los casos donde el valor esperado fue menor a 5). } \\
\text { d Prueba T para muestras independientes. } \\
\text { e } n=53 \text {. } \\
\text { Edad materna de riesgo menor a } 20 \text { y mayor a } 35 \text { años. }\end{array}$} \\
\hline
\end{tabular}


Como era de esperarse, la gravedad de los síntomas de TDAH (figura 1) fue significativamente mayor en los HAR TDAH+ vs. aquellos sin el diagnóstico ( $\mathrm{p}<0.001)$.

En cuanto a la adversidad psicosocial (cuadro 1), se reportó un promedio de 3.3 adversidades (DE=1.4). El 72.6\% $(E S=0.049)$ de los HAR se expuso a tres o más adversidades. En cuanto a las características familiares adversas, el $45.2 \% \quad(E S=0.055)$ de la muestra reportó encontrarse en un ambiente familiar gravemente disfuncional y el $85.7 \%$ $(\mathrm{ES}=0.038)$ reportó discordia marital en sus padres. En el $51.2 \%(\mathrm{ES}=0.055)$ de la muestra se encontró psicopatología en ambos padres y el 67.9\% (ES=0.051) reportó haber completado hasta un grado máximo de estudios de preparatoria completa.

Al comparar el sexo y la edad, además de la adversidad psicosocial, entre aquellos HAR TDAH+ y TDAH- (cuadro 2 ), encontramos que no existieron diferencias significativas entre los grupos, excepto para la presencia de disfunción familiar grave la cual, cuando estuvo presente, incrementó más de 2.5 veces la probabilidad de presentar TDAH en esta población en alto riesgo (IC 95\% 1.06-6.25). Al ajustarse por edad y sexo, este efecto dejó de ser significativo (RM ajustado=2.14 IC 95\% 0.87-5.28). No obstante que el número promedio de adversidades psicosociales fue mayor en los HAR TDAH+ (3.71 DE=1.4) vs. los HAR TDAH- (2.9 DE=1.35; $\mathrm{t}=2.69, \mathrm{gl}=82, \mathrm{p}=0.009$ ), cuando se compararon los grupos por presentar tres o más adversidades psicosociales, no existieron diferencias significativas ( $81.6 \%$ vs. $65.2 \%$; $\mathrm{p}=0.14$ ).

En cuanto a la comparación de otros trastornos psiquiátricos detectados en esta muestra (cuadro 3), encontramos que aunque todos los trastornos evaluados fueron más frecuentes en el reporte de los HAR TDAH+, sólo el trastorno negativista desafiante ( $R M=4.3$, IC 95\%=1.3-14.8) mostró diferencias estadísticamente significativas a favor de los HAR TDAH+, incluso después de ser ajustado por sexo, edad y número de adversidades (RM=2.98; IC 95\%=1.810.9). Aunque el número de otros trastornos psiquiátricos fue significativamente mayor en los HAR TDAH+ (1.9, $\mathrm{DE}=2.2$ vs. 1.1, $\mathrm{DE}=1.02$, respectivamente; $\mathrm{F}=5.24 ; \mathrm{t}=2.12$, $\mathrm{p}<0.02$ ), cuando se compararon los grupos por tener dos o más trastornos psiquiátricos distintos al TDAH, no se alcanzó una diferencia estadísticamente significativa $(47.4 \%$ vs. $34.8 \%, \mathrm{p}=0.271$ ).

Al comparar la frecuencia de casos reportados con deterioro funcional por área (figura 2), encontramos que los padres de HAR TDAH+ reportaron significativamente con mayor frecuencia deterioro en el área de aprendizaje y escuela, incluso después de haber sido ajustado por edad, sexo y número de adversidades (RM=5.09, IC 95\%=1.2820.28). Si bien existió un mayor reporte de deterioro tanto

Cuadro 3. Comparación de los Trastornos Psiquiátricos ${ }^{a}$ actuales entre hermanos en alto riesgo con y sin TDAH ajustados por sexo, edad y número de adversidades

\begin{tabular}{|c|c|c|c|c|c|c|c|}
\hline \multirow[b]{3}{*}{ Trastorno Depresivo Mayor } & \multicolumn{4}{|c|}{$\begin{array}{c}\text { HAR }(n=84) \\
\text { Frecuencia (\%) }\end{array}$} & \multirow{3}{*}{$\frac{\text { Estadístico }^{\mathrm{b}, \mathrm{c}}}{0.698}$} & \multirow{3}{*}{$\frac{p}{0.480}$} & \multirow[b]{2}{*}{$\begin{array}{l}\text { RM no ajustado } \\
\text { (IC al } 95 \%)\end{array}$} \\
\hline & \multicolumn{2}{|c|}{$\begin{array}{l}\text { TDAH } \\
(n=38)\end{array}$} & \multicolumn{2}{|c|}{$\begin{array}{c}\text { Sin TDAH } \\
(n=46)\end{array}$} & & & \\
\hline & 10 & $(26.3)$ & 16 & $(34.8)$ & & & $0.67(0.26-1.72)$ \\
\hline Distimia & 1 & $(2.6)$ & 1 & $(2.2)$ & - & $1.000^{c}$ & $1.22(0.07-20.12)$ \\
\hline Trastorno de ansiedad por separación & 7 & $(18.4)$ & 3 & $(6.5)$ & - & $0.174^{c}$ & $3.24(0.78-13.51)$ \\
\hline Trastorno de ansiedad generalizada & 9 & $(23.7)$ & 14 & $(30.4)$ & 0.477 & 0.624 & $0.71(0.27-1.88)$ \\
\hline Trastorno de angustia & 1 & $(2.6)$ & 1 & $(2.2)$ & - & $1.000^{c}$ & $1.22(0.07-20.12)$ \\
\hline Fobia especifica & 6 & $(15.8)$ & 3 & $(6.5)$ & - & $0.288^{c}$ & $2.69(0.62-11.57)$ \\
\hline Fobia social & 5 & (13.2) & 2 & $(4.3)$ & - & $0.236^{c}$ & $3.33(0.61-18.26)$ \\
\hline Trastorno de estrés postraumático & 2 & (5.3) & & - & - & $0.202^{c}$ & - \\
\hline Trastorno de ansiedad no especificado & 5 & (13.2) & 1 & $(2.2)$ & - & $0.087^{c}$ & $6.82(0.76-61.14)$ \\
\hline Trastorno negativista desafiante & 11 & $(28.9)$ & 4 & (8.7) & - & $0.022^{c}$ & $4.28(1.24-14.82)$ \\
\hline Trastorno de conducta & 5 & $(13.2)$ & 3 & (6.5) & - & $0.458^{c}$ & $2.17(0.48-9.75)$ \\
\hline Trastorno por uso de alcohol & 3 & $(7.9)$ & 2 & $(4.3)$ & - & $0.654^{c}$ & $1.89(0.30-11.91)$ \\
\hline Trastorno por uso de Nicotina & 2 & (5.3) & & - & - & $0.202^{c}$ & - \\
\hline Trastorno por uso de sustancias & 2 & (5.3) & 1 & $(2.2)$ & - & $0.587^{c}$ & $2.50(0.22-28.69)$ \\
\hline Trastornos por tics & 1 & $(2.6)$ & 1 & $(2.2)$ & - & $1.000^{c}$ & $1.22(0.07-20.12)$ \\
\hline Tricotilomanía & 1 & $(2.6)$ & & - & - & $0.452^{c}$ & - \\
\hline Enuresis & 3 & (7.9) & & - & - & $0.089^{c}$ & - \\
\hline Autolesiones sin fin suicidas & 2 & $(5.3)$ & 1 & $(2.2)$ & - & 0.587 & $2.50(0.22-28.69)$ \\
\hline
\end{tabular}




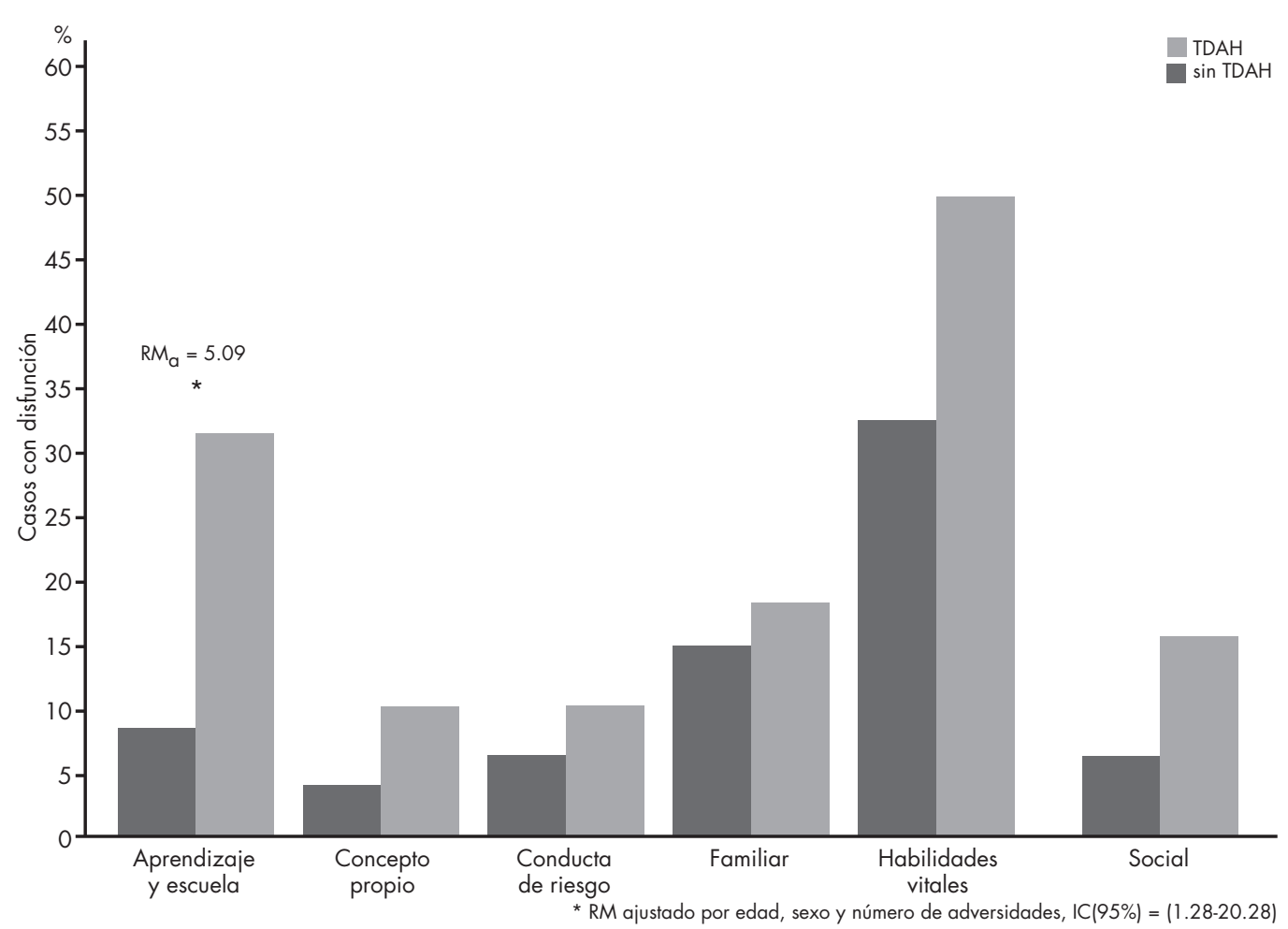

Figura 2. Comparación entre hermanos en alto riesgo con y $\sin T D A H$ en las áreas de deterioro funcional evaluadas por la escala WFIRS-P.

en el área relacionada con el autoconcepto (10.5\% vs. $4.3 \%$, $\mathrm{p}=0.403)$ y el área que se relaciona con el desempeño social (15.8\% vs. $6.5 \%, \mathrm{p}=0.288)$, ninguna alcanzó significancia estadística.

\section{DISCUSIÓN}

El estudio de los hermanos de sujetos con TDAH, los cuales se encuentran también en riesgo para distintos resultados adversos a mediano y largo plazo en su salud mental, nos puede permitir conocer factores de riesgo o vulnerabilidad relacionados con el desarrollo del mismo trastorno o con psicopatología en general, incluso nos puede ayudar a determinar distintos factores protectores relacionados con la resiliencia. Diversos trabajos han mostrado que los hermanos de pacientes con TDAH son un grupo en alto riesgo para distintos desenlaces como desarrollar TDAH u otros trastornos psiquiátricos, tener un funcionamiento más pobre y un menor ajuste psicosocial comparado con hermanos de controles sin TDAH. ${ }^{37,38}$

El objetivo principal de nuestro trabajo fue determinar la frecuencia de TDAH y otros trastornos psiquiátricos en hermanos adolescentes en alto riesgo. En nuestra muestra se encontró que el $45.2 \%$ presentó TDAH, dato similar al reportado por Steinhausen et al., ${ }^{39}$ quienes detectaron que un $47.8 \%$ de los sujetos evaluados tenía TDAH.
Nuestro estudio también encontró un $17.9 \%$ de hermanos que no presentaron trastorno psiquiátrico al momento de la evaluación clínica. Si bien existen trabajos en la bibliografía que reportan la frecuencia de hermanos no afectados con TDAH, ${ }^{18,39}$ en ningún de estos trabajos se especifica si estos sujetos tampoco presentaron otros trastornos psiquiátricos. Este hallazgo es relevante a la luz de la evidencia científica que muestra que, generalmente, este tipo de población con vulnerabilidad biológica compartida se encuentra expuesta a los factores de adversidad psicosocial a los que se exponen los hermanos ya afectados y que acuden a consulta, como pudimos observar en esta muestra de hermanos, lo cual puede incrementar el riesgo de desarrollar cualquier trastorno psiquiátrico, incluido el TDAH. Es indudable que el estudio de sujetos sanos que tienen familiares en primer grado con algún trastorno psiquiátrico como el TDAH, puede ayudar a entender los factores protectores relacionados con la ausencia de trastornos psiquiátricos. ${ }^{40}$

En esta muestra de hermanos en riesgo, casi el $43 \%$ fue del sexo masculino. Otros autores han reportado frecuencias que oscilan entre el $37.3 \%{ }^{39,41}$ y el $54 \% .^{42} \mathrm{Al}$ comprar por sexo entre aquellos que tuvieron TDAH vs. aquellos que no presentaban el trastorno, llama la atención que casi un $42 \%$ de los HAR TDAH+ eran mujeres, además de que no existieron diferencias por sexo entre ambos grupos. Este dato coincide con lo reportado por Biederman et al. ${ }^{43}$ en un estudio donde se evaluaron sujetos no referidos con TDAH, en el cual 
no encontraron diferencias por sexo en cuanto al subtipo de TDAH, la comorbilidad psiquiátrica o la historia de tratamiento. Además, en ese mismo estudio, tanto mujeres como hombres con TDAH mostraron niveles similares en cuanto al funcionamiento cognitivo, psicosocial, escolar y familiar. La falta de diferencias por sexo en cuanto a la proporción de HAR TDAH+ en esta muestra de hermanos adolescentes no referidos, concuerda con revisiones previas ${ }^{44,45}$ las cuales han señalado la preocupación de que solamente las mujeres con un deterioro substancial son referidas para su atención clínica. La investigación sugiere que el sesgo de referencia lleva a subestimar el diagnóstico de TDAH en mujeres, particularmente en las más jóvenes. ${ }^{46}$ Con respecto a la adversidad psicosocial, llama la atención que el 72.6\% de nuestra muestra se encontró expuesta a tres o más adversidades evaluadas. Dentro de los factores de adversidad psicosocial estudiados, resaltan aquellos relacionados con los padres, en especial: 1) la elevada frecuencia en el reporte de discordia marital, 2) los porcentajes elevados de problemas en su salud mental incluida la historia de trastornos por consumo de alcohol y sustancias y 3) el alto porcentaje de baja escolaridad que se encontró en dichos padres. El hallazgo de que una buena proporción de padres presentaron problemas relacionados con su salud mental ha sido ya señalado en otros estudios. Al respecto, Steinhausen et al., ${ }^{47}$ al evaluar a los padres de niños y adolescentes con TDAH, encontraron que en aquellos padres en los cuales persistía el TDAH se observaba mayor severidad en la psicopatología comórbida. En otro reporte previo, Ghanizadeh et al. ${ }^{48}$ señalaron que los trastornos psiquiátricos más frecuentes detectados a lo largo de la vida en los padres, fueron el TDAH (padres $45.8 \%$ vs. madres $17.7 \%$ ) y trastorno depresivo mayor (madres $48.1 \%$ vs. padres $43 \%$ ). El que se presente en los padres algún trastorno psiquiátrico, en especial TDAH, podría incrementar el umbral de reconocimiento para cualquier problema de salud mental en sus hijos, en especial en los menos afectados y de esta manera disminuir la posibilidad de ser atendidos oportunamente. En otras áreas de estudio de la salud mental se ha señalado que cuando el padre tiene un episodio afectivo se afecta el reconocimiento de la psicopatología en sus hijos. ${ }^{49}$ Otros trabajos resaltan la importancia de tratar conjuntamente a los padres que tienen psicopatología, ${ }^{50}$ ya que de no tratarlos se ha demostrado que influyen de manera negativa en el pronóstico a nivel conductual, emocional y social de sus hijos. ${ }^{51}$ En nuestro reporte, los HAR TDAH+ comparados con los HAR TDAH- tuvieron significativamente cinco veces mayor probabilidad de ser reportados por sus padres con afectación en el área escolar y de aprovechamiento. El que los padres no hayan encontrado un motivo suficiente para llevar a estos adolescentes afectados, hermanos de pacientes con TDAH, a consulta, podría explicarse por el efecto mediador de la psicopatología de los padres. Además, la evidencia científica muestra que factores como la presencia de un trastorno externalizado en los sujetos afectados podría incrementar la probabilidad de que sean llevados a atención especializada. ${ }^{44,52}$

La evidencia científica ha señalado la importancia de considerar los distintos factores de adversidad y su relación con distintos trastornos psiquiátricos, principalmente en niños y adolescentes. En nuestro estudio, la disfunción familiar grave incrementó 2.5 veces más la probabilidad de encontrar TDAH en los HAR, hallazgo que perdió significancia cuando se ajustó por sexo y edad. En México, los estudios de Benjet et al. ${ }^{53-55}$ resaltan que los adolescentes mexicanos se encuentran expuestos a distintas adversidades, indicando que es la suma de estos eventos adversos más que su efecto individual lo que explicaría su relación con el inicio de distintos trastornos psiquiátricos. En nuestra muestra el promedio de adversidades encontrado fue por arriba de tres y fue significativamente mayor en los HAR TDAH+ comparados con aquellos que no tuvieron el trastorno; sin embargo no se encontró diferencia cuando se comparó entre aquellos que tenían tres o más adversidades. $\mathrm{Al}$ respecto, Benjet et al. ${ }^{55}$ señalaron en su trabajo que el efecto del número de adversidades encontrado fue no lineal, es decir que aunque la probabilidad de inicio de un trastorno se incrementa con el aumento en el número de adversidades, dicha probabilidad se eleva en un índice decreciente, dicho de otra manera pareciera que después de cierto umbral en la suma de adversidades, el efecto es cada vez menor en la probabilidad de presentar algún trastorno psiquiátrico.

El estudio de esta población de hermanos de pacientes con TDAH, resalta que no solamente se encuentran expuestos al riesgo de desarrollar el mismo trastorno como el sujeto índice, sino también de presentar otros trastornos psiquiátricos como los trastornos del afecto, de ansiedad o de conducta. Excepto para el trastorno negativista desafiante, las frecuencias diagnósticas se mostraron muy similares entre los HAR TDAH+ y los TDAH- cuando se determinaron los trastornos psiquiátricos en el momento actual. Nuestro hallazgo, que muestra que la presencia de TDAH, incluso ajustado por sexo, edad y número de adversidades, en los HAR incrementa casi tres veces la probabilidad de presentar un trastorno negativista desafiante, confirma lo encontrado en otros estudios donde el trastorno negativista desafiante es el trastorno psiquiátrico que con mayor frecuencia se puede encontrar en comorbilidad en sujetos con TDAH. ${ }^{18,48,56}$

Dentro de las limitaciones de nuestro estudio podríamos considerar, primero, que la mayor parte de los adolescentes evaluados provienen de una muestra clínica; segundo, que sólo se evaluaron hermanos adolescentes y no se incluyeron niños; tercero, a pesar de que se hizo más robusto el diagnóstico de los trastornos psiquiátricos a través del consenso, también es cierto que no se pudo eliminar del todo en nuestra muestra el sesgo de recuerdo y, cuarto, el análisis secundario de algunos datos nos sugiere que nuestro estudio podría tener algunos problemas relacionados con la potencia estadística, especialmente para algunos de los resultados secundarios. 
Las fortalezas de nuestro estudio se relacionan con la incorporación a la evaluación de al menos uno de los padres. Los clínicos que participaron en la evaluación eran profesionales con suficiente experiencia clínica en el área de niños y adolescentes, además, la confirmación diagnóstica se determinó por consenso con un psiquiatra certificado, con al menos 15 años de experiencia clínica y, finalmente, la muestra de hermanos adolescentes demostró independencia entre sí, ya que, excepto por un sujeto, el resto provino de familias distintas.

Estos resultados sugieren al clínico que cuando se trata de pacientes con TDAH, el considerar de manera sistemática dentro de la evaluación a los familiares en primer grado, específicamente a los hermanos, es importante ya que se exponen a distintos eventos relacionados con la adversidad psicosocial, manifiestan el mismo trastorno $\mathrm{u}$ otros trastornos psiquiátricos, muestran grados importantes de afectación en distintas áreas del funcionamiento que al no ser tratados muy probablemente afectarán de manera negativa su capacidad de adaptación y funcionamiento a lo largo de la vida. Especial mención merecen los padres de estos sujetos que también muestran porcentajes elevados de trastornos psiquiátricos.

\section{CONCLUSIONES}

Tomando en cuenta el incremento en la probabilidad de tener resultados negativos y riesgos a nivel de salud mental, el estudio clínico de los hermanos de probandos con TDAH, una población en alto riesgo, es necesaria por las distintas implicaciones que tienen a nivel de prevención, atención oportuna y mejora del pronóstico de estos sujetos. La atención de la salud mental de los familiares en primer grado de estos pacientes, sin duda es un factor que no debe de subestimarse ya que de otra manera puede afectar de manera negativa la probabilidad de respuesta a cualquier tratamiento propuesto.

Se requiere realizar más estudios que aborden los factores que protegen y ponen en riesgo, para distintos desenlaces como el TDAH, a los miembros de una familia de sujetos afectados por él.

Estudios subsecuentes deben evaluar, además de los aspectos genéticos, las variables cognitivas, emocionales y de percepción individual del medio ambiente en estas poblaciones, que nos ayuden a entender fenómenos como la vulnerabilidad o la resiliencia a uno o más trastornos psiquiátricos.

\section{AGRADECIMIENTOS}

Los autores de este artículo agradecen el apoyo recibido por la Fundación Miguel Alemán A.C., que contribuyó al financiamiento del proyecto de investigación "Características clínicas y cognitivas que predicen TDAH en hermanos adolescentes en alto riesgo". Así mismo agradecen la participación y las facilidades otorgadas por la Fundación Cultural Federico Hoth A.C. a través de la iniciativa Proyectodah.

\section{REFERENCIAS}

1. American Psychiatric Association. DSM-5 task force. Diagnostic and statistical manual of mental disorders: DSM-5. Quinta edición, Arlington, Va.; 2013.

2. Biederman J, Faraone SV. Attention deficit hyperactivity disorder: a worldwide concern. J Nerv Ment Dis 2004;192(7):453-454.

3. Polanczyk G, De Lima MS, Horta BL, Biederman J et al. The worldwide prevalence of ADHD: a systematic review and metaregression analysis. Am J Psychiatry 2007;164(6):942-948.

4. Benjet C, Borges G, Medina-Mora ME, Zambrano J et al. Youth mental health in a populous city of the developing world: results from the Mexican adolescent mental health survey. J Child Psychol Psychiatry. 2009;50(4):386-395.

5. Catala-Lopez F, Peiro S, Ridao M, Sanfelix-Gimeno G et al. Prevalence of attention deficit hyperactivity disorder among children and adolescents in Spain: a systematic review and meta-analysis of epidemiological studies. BMC Psychiatry 2012;12(1):168.

6. Kessler RC, Adler L, Barkley R et al. The prevalence and correlates of adult ADHD in the United States: results from the National Comorbidity Survey Replication. Am J Psychiatry 2006;163(4):716-723.

7. Faraone SV, Perlis RH, Doyle AE et al. Molecular genetics of attention-deficit/hyperactivity disorder. Biol Psychiatry 2005;57(11):13131323.

8. Burt SA. Rethinking environmental contributions to child and adolescent psychopathology: a meta-analysis of shared environmental influences. Psychol Bull 2009;135(4):608-637.

9. Sprich S, Biederman J, Crawford MH, Mundy E et al. Adoptive and biological families of children and adolescents with ADHD. J Am Acad Child Adolesc Psychiatry 2000;39(11):1432-1437.

10. Biederman J, Faraone SV, Monuteaux MC. Differential effect of environmental adversity by gender: Rutter's index of adversity in a group of boys and girls with and without ADHD. Am J Psychiatry 2002;159(9):1556-1562.

11. Biederman J, Milberger S, Faraone SV et al. Family-environment risk factors for attention-deficit hyperactivity disorder. A test of Rutter's indicators of adversity. Arch Gen Psychiatry 1995;52(6):464-470.

12. Biederman J, Milberger S, Faraone SV et al. Impact of adversity on functioning and comorbidity in children with attention-deficit hyperactivity disorder. J Am Acad Child Adolesc Psychiatry 1995;34(11):1495-1503.

13. Chen W, Zhou $K$, Sham $P$ et al. DSM-IV combined type ADHD shows familial association with sibling trait scores: a sampling strategy for QTL linkage. Am J Med Genet B Neuropsychiatr Genet 2008;147B(8):1450-1460.

14. Faraone SV, Biederman J, Monuteaux MC. Toward guidelines for pedigree selection in genetic studies of attention deficit hyperactivity disorder. Genet Epidemiol 2000;18(1):1-16.

15. Faraone SV. Genetics of adult attention-deficit/hyperactivity disorder. Psychiatr Clin North Am 2004;27(2):303-321.

16. Altink ME, Rommelse NN, Slaats-Willemse DI et al. The dopamine receptor D4 7-repeat allele influences neurocognitive functioning, but this effect is moderated by age and ADHD status: an exploratory study. World J Biol Psychiatry 2012;13(4):293-305.

17. Steinhausen H-C, Zülli-Weilenmann N, Brandeis D, Müller U et al. The behavioural profile of children with attention-deficit/hyperactivity disorder and of their siblings. European Child Adolescent Psychiatry 2012;21(3):157-164.

18. Yang LK, Shang CY, Gau SS. Psychiatric comorbidities in adolescents with attention-deficit hyperactivity disorder and their siblings. Can J Psychiatry 2011;56(5):281-292. 
19. DuPaul GJ, Power TJ, Anastopoulos AD, Reid R. ADHD rating scale-IV: chacklists, norms, and clinical interpretation. New York: Guilford; 1998.

20. DuPaul GJ, Ervin RA, Hook CL, McGoey KE. Peer tutoring for children with attention deficit hyperactivity disorder: effects on classroom behavior and academic performance. J Appl Behav Anal 1998;31(4):579-592.

21. Servera M, Cardo E. [ADHD Rating Scale-IV in a sample of Spanish schoolchildren: normative data and internal consistency for teachers and parents]. Rev Neurol 2007;45(7):393-399.

22. Servera M, Lorenzo-Seva U, Cardo E, Rodriguez-Fornells A et al. Understanding trait and sources effects in attention deficit hyperactivity disorder and oppositional defiant disorder rating scales: mothers', fathers', and teachers' ratings of children from the Balearic Islands. J Clin Child Adolesc Psychol 2010;39(1):1-11.

23. Michanie C, Kunst G, Margulies DS, Yakhkind A. Symptom prevalence of ADHD and ODD in a pediatric population in Argentina. J Atten Disord 2007;11(3):363-367.

24. Goodman R. The Strengths and Difficulties Questionnaire: a research note. J Child Psychol Psychiatry 1997;38(5):581-586.

25. Hawes DJ, Dadds MR. Australian data and psychometric properties of the Strengths and Difficulties Questionnaire. Aust N Z J Psychiatry 2004;38(8):644-651.

26. Koskelainen M, Sourander A, Vauras M. Self-reported strengths and difficulties in a community sample of Finnish adolescents. Eur Child Adolesc Psychiatry 2001;10(3):180-185.

27. Koskelainen M, Sourander A, Kaljonen A. The Strengths and Difficulties Questionnaire among Finnish school-aged children and adolescents. Eur Child Adolesc Psychiatry 2000;9(4):277-284.

28. Woerner W, Becker A, Rothenberger A. Normative data and scale properties of the German parent SDQ. Eur Child Adolesc Psychiatry 2004;13(Suppl 2)II:3-10.

29. Bourdon KH, Goodman R, Rae DS, Simpson G et al. The Strengths and Difficulties Questionnaire: U.S. normative data and psychometric properties. J Am Acad Child Adolesc Psychiatry 2005;44(6):557-564.

30. Fabregat-Ferrer E, MJ P-G. Cribado de problemas psicosociales en Atención Primaria. Rev Pediatr Aten Primaria 2009(11):425-437.

31. Maziade M, Rouleau N, Lee B, Rogers A et al. Atomoxetine and neuropsychological function in children with attention-deficit/hyperactivity disorder: results of a pilot study. J Child Adolesc Psychopharmacol 2009;19(6):709-718.

32. Shaffer D, Gould MS, Brasic J et al. A children's global assessment scale (CGAS). Arch Gen Psychiatry 1983;40(11):1228-1231.

33. Sheehan DV, Lecrubier $Y$, Sheehan KH et al. The Mini-International Neuropsychiatric Interview (M.I.N.I.): the development and validation of a structured diagnostic psychiatric interview for DSM-IV and ICD-10. J Clin Psychiatry 1998;59(Suppl 20):22-33; quiz 34-57.

34. Amorim P, Lecrubier Y, Weiller E, Hergueta T et al. DSM-IH-R Psychotic Disorders: procedural validity of the Mini International Neuropsychiatric Interview (MINI). Concordance and causes for discordance with the CIDI. Eur Psychiatry. 1998;13(1):26-34.

35. Smilkstein G. The family APGAR: a proposal for a family function test and its use by physicians. J Fam Pract 1978;6(6):1231-1239.

36. Smilkstein G, Ashworth C, Montano D. Validity and reliability of the family APGAR as a test of family function. J Fam Pract 1982;15(2):303-311.

37. Faraone SV, Biederman J, Mennin D, Gershon J et al. A prospective four-year follow-up study of children at risk for ADHD: psychiatric, neuropsychological, and psychosocial outcome. J American Academy Child Adolescent Psychiatry 1996;35(11):1449-1459.
38. Seidman LJ, Biederman J, Monuteaux MC, Weber W et al. Neuropsychological functioning in nonreferred siblings of children with attention deficit/hyperactivity disorder. J Abnorm Psychol 2000;109(2):252-265.

39. Steinhausen HC, Zülli-Weilenmann N, Brandeis D, Müller UC et al. The behavioural profile of children with attention-deficit/hyperactivity disorder and of their siblings. Eur Child Adolesc Psychiatry 2012.

40. Modesto-Lowe V, Yelunina L, Hanjan K. Attention-deficit/hyperactivity disorder: a shift toward resilience? Clin Pediatr (Phila) 2011;50(6):518-524.

41. Rommelse NN, Oosterlaan J, Buitelaar J, Faraone SV, Sergeant JA. Time reproduction in children with ADHD and their nonaffected siblings. J Am Acad Child Adolesc Psychiatry 2007;46(5):582-590.

42. Milberger S, Biederman J, Faraone SV, Chen L et al. Further evidence of an association between attention-deficit/hyperactivity disorder and cigarette smoking. Findings from a high-risk sample of siblings. Am J Addict 1997;6(3):205-217.

43. Biederman J, Kwon A, Aleardi M et al. Absence of gender effects on attention deficit hyperactivity disorder: findings in nonreferred subjects. Am J Psychiatry 2005;162(6):1083-1089.

44. Rucklidge JJ. Gender differences in attention-deficit/hyperactivity disorder. Psychiatr Clin North Am 2010;33(2):357-373.

45. Hinshaw SP. Preadolescent girls with attention-deficit/hyperactivity disorder: I. Background characteristics, comorbidity, cognitive and social functioning, and parenting practices. J Consult Clin Psychol 2002;70(5):1086-1098.

46. Gershon J. A meta-analytic review of gender differences in ADHD. J Atten Disord 2002;5(3):143-154.

47. Steinhausen HC, Göllner J, Brandeis D, Müller UC et al. Psychopathology and personality in parents of children with ADHD. J Atten Disord. Mar 2012.

48. Ghanizadeh A, Mohammadi MR, Moini R. Comorbidity of psychiatric disorders and parental psychiatric disorders in a sample of Iranian children with ADHD. J Atten Disord 2008;12(2):149-155.

49. Maoz H, Goldstein T, Goldstein BI et al. The effects of parental mood on reports of their children's psychopathology. J Am Acad Child Adolesc Psychiatry 2014;53(10):1111-1122.e1115.

50. Vidair HB, Reyes JA, Shen $S$ et al. Screening parents during child evaluations: exploring parent and child psychopathology in the same clinic. J Am Acad Child Adolesc Psychiatry 2011;50(5):441-450.

51. Breaux RP, Harvey EA, Lugo-Candelas CI. The role of parent psychopathology in the development of preschool children with behavior problems. J Clin Child Adolesc Psychol 2014;43(5):777-790.

52. Biederman J, Faraone SV, Keenan $\mathrm{K}$ et al. Further evidence for family-genetic risk factors in attention deficit hyperactivity disorder. Patterns of comorbidity in probands and relatives psychiatrically and pediatrically referred samples. Arch Gen Psychiatry 1992;49(9):728-738.

53. Benjet C, Borges G, Medina-Mora ME, Zambrano J et al. Descriptive epidemiology of chronic childhood adversity in Mexican adolescents. J Adolesc Health 2009;45(5):483-489.

54. Benjet C. Childhood adversities of populations living in low-income countries: prevalence, characteristics, and mental health consequences. Curr Opin Psychiatry 2010;23(4):356-362.

55. Benjet C, Borges G, Medina-Mora ME. Chronic childhood adversity and onset of psychopathology during three life stages: childhood, adolescence and adulthood. J Psychiatr Res 2010;44(11):732-740.

56. Ghanizadeh A. Psychiatric comorbidity differences in clinic-referred children and adolescents with ADHD according to the subtypes and gender. J Child Neurol 2009;24(6):679-684. 